\title{
Numerical approximation for a time optimal control problems governed by semi-linear heat equations
}

\author{
Guojie Zheng ${ }^{1,2^{*}}$ and Jingben Yin ${ }^{3}$
}

\section{"Correspondence:}

guojiezheng@yeah.net

${ }^{1}$ College of Mathematics and

Information Science, Henan Normal

University, Xinxiang, 453007,

P.R. China

${ }^{2}$ School of Computational and Applied Mathematics, University of

the Witwatersrand, Wits 2050

Johannesburg, South Africa

Full list of author information is

available at the end of the article

\begin{abstract}
In this paper, we study the optimal time for a time optimal control problem $(\mathcal{P})$, governed by an internally controlled semi-linear heat equation. By projecting the original problem via the finite element method, we obtain another time optimal control problem $\left(\mathcal{P}_{h}\right)$ governed by a semi-linear system of ordinary differential equations. Here, $h$ is the mesh sizes of the finite element spaces. The purpose of this study is to approach the optimal time for the problem $(\mathcal{P})$ through the optimal time for the problem $\left(\mathcal{P}_{h}\right)$. We obtain error estimates between the optimal times in terms of $h$.
\end{abstract}

MSC: $35 \mathrm{~K} 05 ; 49 J 20$

Keywords: heat equation; time optimal control; finite element methods; numerical approximation

\section{Introduction}

One of the most important optimal control problems is how to drive the corresponding trajectory of the equation from an initial state to a given target set in the shortest time, through applying constrained controllers. With regard to this kind of problems, the optimal time, is a very significant value. In this paper, we study numerical approximation for a time optimal control problems governed by semi-linear heat equations. We first project the problem into another time optimal control problem of ordinary differential equations, via the finite element method. Then, we establish error estimates between the optimal times for the original problem and its projected problem.

Let us first state the time optimal control problem $(\mathcal{P})$ studied in this paper. We begin with introducing the controlled equation. Let $\Omega$ be a convex and bounded domain, with smooth boundary $\partial \Omega$, in $\mathbb{R}^{d}(d=1,2,3)$. Let $\omega$ be an open and nonempty subset of $\Omega$. In this paper, we consider the following semi-linear controlled heat equation:

$$
\begin{cases}\partial_{t} y(x, t)-\Delta y(x, t)=f(y(x, t))+\chi_{\omega} u(x, t) & \text { in } \Omega \times(0,+\infty), \\ y(x, t)=0 & \text { on } \partial \Omega \times(0,+\infty), \\ y(x, 0)=y_{0}(x) & \text { in } \Omega,\end{cases}
$$

where the initial value $y_{0}$ belongs to $H_{0}^{1}(\Omega) \cap L^{\infty}(\Omega)$, and $u(\cdot)$ is a control function taken from the space $L^{\infty}\left(0,+\infty ; L^{2}(\Omega)\right)$, and $f(\cdot)$ is a $C^{1}$ function from $\mathbb{R}$ to $\mathbb{R}$. We assume that

$$
\left|f^{\prime}(x)\right| \leq L \quad \text { for } x \in \mathbb{R}
$$

O2014 Zheng and Yin; licensee Springer. This is an Open Access article distributed under the terms of the Creative Commons Attribution License (http://creativecommons.org/licenses/by/2.0), which permits unrestricted use, distribution, and reproduction in any medium, provided the original work is properly cited. 
and

$$
f(0)=0
$$

It is easy to see that under the present assumptions this semi-linear heat equations has a unique solution (see $[1,2])$. Throughout this paper, we will treat the solutions of (1.1) as functions of the time variable $t$, from $\mathbb{R}^{+} \equiv[0,+\infty)$ to the space $L^{2}(\Omega)$, and denote $y\left(\cdot ; y_{0}, u\right)$ the unique solution of (1.1) corresponding to the control $u$ and the initial value $y_{0}$. We denote $\|\cdot\|$ and $\langle\cdot, \cdot\rangle$ to the usual norm and the inner product of $L^{2}(\Omega)$ respectively. Besides, variables $x$ and $t$ for functions of $(x, t)$ and variable $x$ for functions of $x$ will be omitted, provided that it is not going to cause any confusion. The constraint control set is taken as

$$
\mathcal{U}_{a d}=\left\{v \in L^{\infty}\left(0,+\infty ; L^{2}(\Omega)\right) ;\|v(t)\|_{L^{2}(\Omega)} \leq 1 \text { for almost every } t \in[0,+\infty)\right\}
$$

while the target set is the closed ball $B(0,1) \equiv\left\{w \in L^{2}(\Omega) ;\|w\| \leq 1\right\}$. The time optimal control problem reads as follows:

$$
(\mathcal{P}): \min _{u \in \mathcal{U}_{a d}}\left\{T ; y\left(T ; y_{0}, u\right) \in B(0,1)\right\}
$$

In this problem, the number $T^{*}\left(y_{0}\right)=\min _{u \in \mathcal{U}_{a d}}\left\{T ; y\left(T ; y_{0}, u\right) \in B(0,1)\right\}$ is called the optimal time, while a control $u^{*}$, in the set $\mathcal{U}_{a d}$, and holding the property that $y\left(T^{*}\left(y_{0}\right) ; y_{0}, u^{*}\right) \in$ $B(0,1)$, is called an optimal control. For each $y_{0} \in L^{2}(\Omega)$, we define $T^{*}\left(y_{0}\right)$ to be the optimal time for the problem $(\mathcal{P})$. Thus, $T^{*}(\cdot)$ is a function from $L^{2}(\Omega)$ to $\mathbb{R}^{+}$.

We next build the approximate problem for $(\mathcal{P})$. We first build a finite element space $V_{0}^{h}$, which will be further discussed in the next section. Let $P_{h}$ be the $L^{2}$-projection from $L^{2}(\Omega)$ to $V_{0}^{h}$, and we project the target set $B(0,1)$ into

$$
B_{h}(0,1) \equiv\left\{w_{h} \in V_{0}^{h} ;\left\|w_{h}\right\| \leq 1\right\} .
$$

Now, we study the following semi-discrete system:

$$
\left\{\begin{array}{l}
\left\langle y_{h}^{\prime}(t), v_{h}\right\rangle+\left\langle\nabla y_{h}(t), \nabla v_{h}\right\rangle=\left\langle f\left(y_{h}\right), v_{h}\right\rangle+\left\langle\chi_{\omega} u, v_{h}\right\rangle, \quad \forall v_{h} \in V_{0}^{h}, t \geq 0 \\
y_{h}(0)=P_{h} y_{0} .
\end{array}\right.
$$

Here, the control $u(\cdot)$ is taken from the constraint control set $\mathcal{U}_{a d}$. We denote $y_{h}\left(\cdot ; P_{h} y_{0}, u\right)$ the solution of (1.4) corresponding to the control $u$ and the initial value $P_{h} y_{0}$. Consequently, we project the problem $(\mathcal{P})$ into the following time optimal control problem of ordinary differential equations:

$$
\left(\mathcal{P}_{h}\right): \min _{u \in \mathcal{U}_{a d}}\left\{T ; y_{h}\left(T ; P_{h} y_{0}, u\right) \in B_{h}(0,1)\right\}
$$

For each $y_{0}^{h} \in V_{0}^{h}$, we define $T_{h}^{*}\left(y_{0}^{h}\right)$ to be the optimal time for the problem $\left(\mathcal{P}_{h}\right)$ where the initial value $P_{h} y_{0}$ is replaced by $y_{0}^{h}$. Thus, $T_{h}^{*}(\cdot)$ is a function from $V_{0}^{h}$ to $\mathbb{R}^{+}$, and $T_{h}^{*}\left(P_{h} y_{0}\right)$ is the optimal time for $\left(\mathcal{P}_{h}\right)$.

In this study, we derive the error estimates between $T^{*}\left(y_{0}\right)$ and $T_{h}^{*}\left(P_{h} y_{0}\right)$, in terms of $h$. The main results of the paper are presented as follows. 
Theorem 1.1 Let $y_{0} \in H_{0}^{1}(\Omega) \cap L^{\infty}(\Omega)$. Equations (1.2) and (1.3) hold, and the constant $L$ in (1.2) satisfies $L<\lambda_{1}$. Then there exists a positive number $h_{0}$ such that

$$
\left|T_{h}^{*}\left(P_{h} y_{0}\right)-T^{*}\left(y_{0}\right)\right| \leq C h, \quad \text { when } 0<h<h_{0} .
$$

Here and throughout the rest of the paper, $\lambda_{1}$ stand for the first eigenvalue of the operator $-\triangle$, with the Dirichlet boundary condition, and $C$ stands for a positive constant independent of $h$. This constant varies in different contexts.

Since $(\mathcal{P})$ is an optimal control problem governed by an infinite dimensional system, while $\left(\mathcal{P}_{h}\right)$ is an optimal control problem governed by a finite dimensional system, the study of $T^{*}\left(y_{0}\right)$ should be much more difficult than that of $T_{h}^{*}\left(P_{h} y_{0}\right)$. The main purpose of this paper is to study the approximation of $T^{*}\left(y_{0}\right)$ through $T_{h}^{*}\left(P_{h} y_{0}\right)$. This kind of problem has only been addressed in quite limited papers. To the best of our knowledge, the first study on this subject is the paper [3]. In this [3], the author was concerned with time optimal control problems for a class of boundary scalar controlled linear parabolic equations, obtained error estimates for optimal times, presented a full discretization of the original problem followed by numerical tests. In our paper, the problem which we study is governed by the internally controlled semi-linear heat equation. The other important literature on this subject which we would like to mention is $[4,5]$.

The rest of the paper is structured as follows. In Section 2, we first construct finite element spaces $V_{0}^{h}$, then give certain properties for the functions $T^{*}(\cdot)$ and $T_{h}^{*}(\cdot)$. Section 3 presents the proof of Theorem 1.1.

\section{Finite element spaces $V_{0}^{h}$ and preliminary results}

Since $\Omega$ is a convex set with a smooth boundary, there exists a positive number

$$
h_{0} \equiv h_{0}(\Omega) \quad \text { (depending only on } \Omega \text { ) }
$$

having the property: corresponding to each $h$, with $0<h<h_{0}$, one can construct such a family $\mathcal{T}^{h}$ of regular triangulations in $\bar{\Omega}$ that satisfies the following conditions (see [6]):

$\left(\mathrm{A}_{1}\right)$ There exist two positive constants $\rho$ and $\sigma$ independent of $h$, such that $\rho(\tau) / \sigma(\tau) \leq \sigma$ and $h / \rho(\tau) \leq \rho$ for each element $\tau$ in $\mathcal{T}^{h}$. (The notations $\rho(\tau)$ and $\sigma(\tau)$ stand for the diameter of the set $\tau$ and the diameter of the greatest ball contained in $\tau$, respectively.)

$\left(\mathrm{A}_{2}\right) \bar{\Omega}_{h} \equiv \bigcup_{\tau \in \mathcal{T}^{h}} \tau$ is a polygonal approximation of $\bar{\Omega}$. The vertices of $\mathcal{T}^{h}$, which are on the boundary $\partial \Omega_{h}$, belong to $\partial \Omega$. Furthermore, we see that the measure of $\left(\Omega \backslash \Omega_{h}\right) \leq C h^{2}$.

For each $\tau \in \mathcal{T}^{h}$, we denote $\mathcal{S}(\tau)$ to the space of all polynomials of 1-order and defined on $\tau$. Corresponding to the state space $L^{2}(\Omega)$, we build a finite element space as follows:

$$
V_{0}^{h}=\left\{v_{h} \in C(\bar{\Omega}) ;\left.v_{h}\right|_{\tau} \in \mathcal{S}(\tau) \text { for every } \tau \in \mathcal{T}^{h} \text { and }\left.v_{h}\right|_{\bar{\Omega} \backslash \Omega_{h}}=0\right\}
$$

It is a subspace of $H_{0}^{1}(\Omega)$. Let $P_{h}$ be the $L^{2}$-projection from $L^{2}(\Omega)$ to $V_{0}^{h}$, namely,

$$
\left\langle P_{h} v, v_{h}\right\rangle=\left\langle v, v_{h}\right\rangle, \quad \forall v \in L^{2}(\Omega), v_{h} \in V_{0}^{h} .
$$

Now, we will present some lemmas, which will be used later. 
Lemma 2.1 Suppose (1.2) and (1.3) hold, and $y_{0} \in H_{0}^{1}(\Omega) \cap L^{\infty}(\Omega)$. Then the corresponding solution $y\left(\cdot ; y_{0}, u\right)$ of $(1.1)$ is global and the following inequality holds:

$$
\left\|y\left(t ; y_{0}, 0\right)\right\| \leq\left\|y_{0}\right\| e^{-\left(\lambda_{1}-L\right) t} \quad \text { for } t \geq 0
$$

Proof The proof for the existence of the global solution for (1.1) can be viewed in [1]. Now, we are going to prove inequality (2.2). According to (1.2) and (1.3), we get

$$
|f(y)| \leq L|y|
$$

Let $F(t)=\int_{\Omega}\left|y\left(t ; y_{0}, 0\right)\right|^{2} d t$, for $t \in[0,+\infty)$. Then,

$$
\begin{aligned}
F^{\prime}(t) & \leq-2 \lambda_{1} F(t)+2 \int_{\Omega}\left|y\left(t ; y_{0}, 0\right) f\left(y\left(t ; y_{0}, 0\right)\right)\right| d x \\
& \leq-2 \lambda_{1} F(t)+2 L \int_{\Omega}\left|y\left(t ; y_{0}, 0\right)\right|^{2} d x \\
& \leq-2\left(\lambda_{1}-L\right) F(t) .
\end{aligned}
$$

From this, we can complete the proof of the lemma.

Remark 2.1 With the same argument, we can also derive that the solution $y_{h}\left(\cdot ; y_{0}^{h}, 0\right)$ of (1.4) also satisfies the following inequality:

$$
\left\|y_{h}\left(t ; y_{0}^{h}, 0\right)\right\| \leq\left\|y_{0}^{h}\right\| e^{-\left(\lambda_{1}-L\right) t} \quad \text { for } t \geq 0 .
$$

Lemma 2.2 Suppose $y_{0} \in H_{0}^{1}(\Omega) \cap L^{\infty}(\Omega)$ and $u \in \mathcal{U}_{\text {ad }}$. Then, for each $T>0$, there exists a constant $C_{T}$, which is independent of h but depends on $T$, such that

$$
\left\|y\left(\cdot ; y_{0}, u\right)-y_{h}\left(\cdot ; P_{h} y_{0}, u\right)\right\|_{C\left([0, T] ; L^{2}(\Omega)\right)} \leq C_{T} h\left(\left\|y_{0}\right\|_{1}+\|u(t)\|_{L^{2}\left(0, T ; L^{2}(\Omega)\right)}\right) .
$$

We can deduce this lemma by classical finite element analysis; see [7] and [8].

Lemma 2.3 Suppose that $L<\lambda_{1}$, and let $g$ be the function from $\mathbb{R}^{+}$to $\mathbb{R}^{+}$defined by

$$
g(s)= \begin{cases}0, & s \in[0,1], \\ \frac{1}{\lambda_{1}-L} \ln s, & s \in(1,+\infty) .\end{cases}
$$

Then, we have

$$
T^{*}\left(y_{0}\right) \leq g\left(\left\|y_{0}\right\|\right) \quad \text { for all } y_{0} \in L^{2}(\Omega)
$$

and

$$
T_{h}^{*}\left(y_{0}^{h}\right) \leq g\left(\left\|y_{0}^{h}\right\|\right) \quad \text { for all } y_{0}^{h} \in V_{0}^{h} .
$$

Proof Clearly, it suffices to show that the desired inequality in this lemma stands in the case that $y_{0} \notin B(0,1)$. According to Lemma 2.1, we observe that the solution $y\left(\cdot ; y_{0}, 0\right)$ of 
(1.1) with $u=0$, has the estimate

$$
\left\|y\left(t ; y_{0}, 0\right)\right\| \leq e^{-\left(\lambda_{1}-L\right) t}\left\|y_{0}\right\| \quad \text { for each } t \geq 0
$$

Combined with $L<\lambda_{1}$, we see that when $t=\frac{1}{\lambda_{1}-L} \ln \left\|y_{0}\right\|$,

$$
\left\|y\left(t ; y_{0}, 0\right)\right\| \leq e^{-\left(\lambda_{1}-L\right) \frac{1}{\lambda_{1}-L} \ln \left\|y_{0}\right\|}\left\|y_{0}\right\|=1
$$

Namely, $y\left(\cdot ; y_{0}, 0\right)$ have entered into the ball $B(0,1)$ at time $t=\frac{1}{\lambda_{1}-L} \ln \left\|y_{0}\right\|$. This fact, together with the optimality of $T^{*}\left(y_{0}\right)$ to the problem $(\mathcal{P})$, yields the inequality:

$$
T^{*}\left(y_{0}\right) \leq \frac{1}{\lambda_{1}-L} \ln \left\|y_{0}\right\| .
$$

Thus, we obtain the estimate (2.6). With the same argument, we can also obtain inequality (2.7). This completes the proof of the lemma.

\section{The proof of Theorem 1.1}

Let $h_{0}$ be the positive number given in (2.1). It suffices to show that the following two inequalities hold for any $h$ with $0<h<h_{0}$ :

$$
T_{h}^{*}\left(P_{h} y_{0}\right)-T^{*}\left(y_{0}\right) \leq C h
$$

and

$$
T^{*}\left(y_{0}\right)-T_{h}^{*}\left(P_{h} y_{0}\right) \leq C h
$$

We first prove the inequality (3.1). It is well knows that there exist optimal controls for problem $(\mathcal{P})$ and $\left(\mathcal{P}_{h}\right)$, respectively (see $[9,10]$ and [11]). Let $u^{*}$ be the optimal control to the problem $(\mathcal{P})$. Then, by $(2.5)$ we obtain

$$
\left\|y\left(T^{*}\left(y_{0}\right) ; y_{0}, u^{*}\right)-y_{h}\left(T^{*}\left(y_{0}\right) ; P_{h} y_{0}, u^{*}\right)\right\| \leq C h .
$$

From the optimality of $T^{*}\left(y_{0}\right)$ and $u^{*}$ to the problem $(\mathcal{P})$, it follows that

$$
\left\|y\left(T^{*}\left(y_{0}\right) ; y_{0}, u^{*}\right)\right\|=1
$$

Along with the above-mentioned inequality, this indicates that

$$
\left\|y_{h}\left(T^{*}\left(y_{0}\right) ; P_{h} y_{0}, u^{*}\right)\right\| \leq 1+C h
$$

Write $z_{h}=y_{h}\left(T^{*}\left(y_{0}\right) ; P_{h} y_{0}, u^{*}\right)$. There are only two possibilities: $z_{h}$ either belongs to $B_{h}(0,1)$ or is outside of $B_{h}(0,1)$.

In the first case, by the optimality of $T_{h}^{*}\left(P_{h} y_{0}\right)$ to the problem $\left(\mathcal{P}_{h}\right)$, we deduce that $T_{h}^{*}\left(P_{h} y_{0}\right) \leq T^{*}\left(y_{0}\right)$. Therefore, the inequality (3.1) holds for this case.

In the second case, we let $T_{h}^{*}\left(z_{h}\right)$ and $w_{h}^{*}$ be the optimal time and an optimal control to the problem $\left(\mathcal{P}_{h}\right)$, where the initial state $P_{h} y_{0}$ is replaced by the state $z_{h}$. (The existence of 
such an optimal control can be verified easily.) Then, the solution $y_{h}\left(\cdot ; z_{h}, w_{h}^{*}\right)$ takes value in $B_{h}(0,1)$ at time $T_{h}^{*}\left(z_{h}\right)$. One can utilize Lemma 2.3 and (3.3) to deduce that

$$
T_{h}^{*}\left(z_{h}\right) \leq \frac{1}{\lambda_{1}-L} \ln \left\|z_{h}\right\| \leq \frac{1}{\lambda_{1}-L} \ln (1+C h) \leq C h .
$$

Now we construct another control $\bar{u}_{h}$ by setting

$$
\bar{u}_{h}(t)= \begin{cases}u^{*}(t), & t \in\left[0, T^{*}\left(y_{0}\right)\right], \\ w_{h}^{*}\left(t-T^{*}\left(y_{0}\right)\right), & t \in\left(T^{*}\left(y_{0}\right),+\infty\right) .\end{cases}
$$

Clearly, $\bar{u}_{h} \in \mathcal{U}_{a d}$, and the solution $y_{h}\left(\cdot ; P_{h} y_{0}, \bar{u}_{h}\right)$ takes value in $B_{h}(0,1)$ at time $T^{*}\left(y_{0}\right)+$ $T_{h}^{*}\left(z_{h}\right)$. Combined with the optimality of $T_{h}^{*}\left(P_{h} y_{0}\right)$ to the problem $\left(\mathcal{P}_{h}\right)$, these indicate that

$$
T_{h}^{*}\left(P_{h} y_{0}\right) \leq T^{*}\left(y_{0}\right)+T_{h}^{*}\left(z_{h}\right)
$$

This inequality, together with (3.4), yields the estimate (3.1) for the second case. In summary, we conclude that the estimate (3.1) stands.

Next, we are in the position to prove (3.2). Let $u_{h}^{*}$ be the optimal control to the problem $\left(\mathcal{P}_{h}\right)$. Then it follows from $(2.5)$ that

$$
\left\|y\left(T_{h}^{*}\left(P_{h} y_{0}\right) ; y_{0}, u_{h}^{*}\right)-y_{h}\left(T_{h}^{*}\left(P_{h} y_{0}\right) ; P_{h} y_{0}, u_{h}^{*}\right)\right\| \leq C h .
$$

By the optimality of $T_{h}^{*}\left(P_{h} y_{0}\right)$ and $u_{h}^{*}$ to the problem $\left(\mathcal{P}_{h}\right)$, we get

$$
\left\|y_{h}\left(T_{h}^{*}\left(P_{h} y_{0}\right) ; P_{h} y_{0}, u_{h}^{*}\right)\right\|=1 .
$$

Therefore, we have

$$
\left\|y\left(T_{h}^{*}\left(P_{h} y_{0}\right) ; y_{0}, u_{h}^{*}\right)\right\| \leq 1+C h .
$$

Write $z=y\left(T_{h}^{*}\left(P_{h} y_{0}\right) ; y_{0}, u_{h}^{*}\right)$. There are only two possibilities: $z$ either belongs to $B(0,1)$ or is outside of $B(0,1)$.

In the first case, the solution $y\left(T_{h}^{*}\left(P_{h} y_{0}\right) ; y_{0}, u_{h}^{*}\right)$ takes value in $B(0,1)$ at time $T_{h}^{*}\left(P_{h} y_{0}\right)$. This, together with the optimality of $T^{*}\left(y_{0}\right)$ to the problem $(\mathcal{P})$, indicates that $T^{*}\left(y_{0}\right) \leq$ $T_{h}^{*}\left(P_{h} y_{0}\right)$. Therefore, the inequality (3.2) stands in the first case.

In the second case, we let $T^{*}(z)$ and $w^{*}$ be the optimal time and an optimal control to the problem $(\mathcal{P})$, where $y_{0}$ is replaced by $z$.

Then, the solution $y\left(\cdot ; z, w^{*}\right)$ takes value in the $\operatorname{target}$ set $B(0,1)$ at time $T^{*}(z)$. Furthermore, it follows from Lemma 2.3 and (3.5) that

$$
T^{*}(z) \leq \frac{1}{\lambda_{1}-L} \ln \|z\| \leq \frac{1}{\lambda_{1}-L} \ln (1+C h) \leq C h .
$$

Now we construct another control $\bar{u}$ by setting

$$
\bar{u}(t)= \begin{cases}u_{h}^{*}(t), & t \in\left[0, T_{h}^{*}\left(P_{h} y_{0}\right)\right], \\ w^{*}\left(t-T_{h}^{*}\left(P_{h} y_{0}\right)\right), & t \in\left(T_{h}^{*}\left(P_{h} y_{0}\right),+\infty\right) .\end{cases}
$$


Clearly, $\bar{u} \in \mathcal{U}_{a d}$, and the solution $y\left(\cdot ; y_{0}, \bar{u}\right)$ takes value in $B(0,1)$ at time $T_{h}^{*}\left(P_{h} y_{0}\right)+T^{*}(z)$. Combined with the optimality of $T^{*}\left(y_{0}\right)$ to the problem $(\mathcal{P})$, these indicate that

$$
T^{*}\left(y_{0}\right) \leq T_{h}^{*}\left(P_{h} y_{0}\right)+T^{*}(z)
$$

This inequality, together with (3.6), gives the estimate (3.2) for the second case. In summary, we conclude that the estimate (3.2) stands, and we can complete the proof of this theorem.

\section{Competing interests}

The authors declare that they have no competing interests.

\section{Authors' contributions}

GZ provided the question. GZ and JY gave the proof for the main result together. All authors read and approved the final manuscript.

\section{Author details}

${ }^{1}$ College of Mathematics and Information Science, Henan Normal University, Xinxiang, 453007, P.R. China. ${ }^{2}$ School of Computational and Applied Mathematics, University of the Witwatersrand, Wits 2050, Johannesburg, South Africa. ${ }^{3}$ Department of Mathematics, Henan Institute of Science and Technology, Xinxiang, 453003, P.R. China.

\section{Acknowledgements}

The authors would like to express their sincere thanks to the referees for their providing several important references and for their valuable suggestions. This work was partially supported by the National Natural Science Foundation of China under Grants (U1204105, 61203293), the Natural Science Foundation of Zhejiang (Y61 10751), the Natural Science Foundation of Ningbo (2010A610096), the Key Foundation of Henan Educational Committee (13A120524, 12B120006), and the National Research Foundation of South Africa.

\section{Received: 25 December 2013 Accepted: 4 March 2014 Published: 20 Mar 2014}

\section{References}

1. Cazenave, T, Haraux, A: An Introduction to Semilinear Evolution Equations. Clarendon, Oxford (1998)

2. Evans, LC: Partial Differential Equation. Graduate Studies in Mathematics, vol. 19. Am. Math. Soc., Providence (1998)

3. Knowles, G: Finite element approximation of parabolic time optimal control problem. SIAM J. Control Optim. 20, 414-427 (1982)

4. Wang, G, Yu, X: Error estimates for an optimal control problem governed by the heat equation with state and control constraints. Int. J. Numer. Anal. Model. 7, 30-65 (2010)

5. Wang, G, Zheng, G: An approach to the optimal time for a time optimal control problem of an internally controlled heat equation. SIAM J. Control Optim. 50, 601-628 (2012)

6. Ciarlet, P: The Finite Element Method for Elliptic Problems. North-Holland, Amsterdam (1978)

7. Chrysafinos, K, Hou, LS: Error estimates for semidiscrete finite element approximations of linear and semilinear parabolic equations under minimal regularity assumptions. SIAM J. Numer. Anal. 40, 282-306 (2002)

8. Thomée, V: Galerkin Finite Element Methods for Parabolic Equations. Springer, Berlin (1997)

9. Barbu, V: Analysis and Control of Nonlinear Infinite Dimensional Systems. Academic Press, New York (1993)

10. Li, X, Yong, J: Optimal Control Theory for Infinite Dimensional Systems. Birkhäuser Boston, Cambridge (1995)

11. Wang, G: $L^{\infty}$-Null controllability for the heat equation and its consequences for the time optimal control problem. SIAM J. Control Optim. 47, 1701-1720 (2008) 